\title{
Coherent Nanowhiskerography
}

\author{
S. N. Maksimovsky ${ }^{1,2}$, A. Yu. Stavtsev ${ }^{2} \&$ V. N. Ivanova ${ }^{2}$ \\ ${ }^{1}$ P. N. Lebedev Physical Institute of the Russian Academy of Sciences, Russia \\ ${ }^{2}$ Federal State Budget Educational Institution of Higher Education "K.G. Razumovsky Moscow State University \\ of technologies and management (the First Cossack University)", Russia \\ Correspondence: S. N. Maksimovsky, P. N. Lebedev Physical Institute of the Russian Academy of Sciences, \\ Russia. E-mail: maximovsky.sergej@yandex.ru
}

Received: March 26, 2019

Accepted: April 10, 2019

Online Published: April 30, 2019

doi: $10.5539 /$ jmsr.v8n2p26

URL: https://doi.org/10.5539/jmsr.v8n2p26

\begin{abstract}
A new technology is suggested for crystallizing arbitrary materials at temperatures of $3000-4000^{\circ} \mathrm{C}$ and pressures of 20-100 thousand atmospheres. First experimental results were obtained in a foil-coated polycarbonate that comprised diffraction gratings.

It was established that a laser beam reflected from the diffraction gratings is self-focused inside polycarbonate and becomes a seed, which changes the polycarbonate structure and leads to vitrification and growth of ordered structures from symmetrically arranged whiskers. This results in a light dispersion effect in composites. The results concerning whiskers formed with sharp tips are presented.
\end{abstract}

We used data obtained in these experiments for developing a model of crystallization with the rates of 80-100 $\mathrm{m} / \mathrm{s}$. In this case, not only crystals grow, but nanowhiskers also. A new phenomenon of nanowhiskerography was discovered, which can be used for developing the defence against falsification that cannot be reproduced by polygraphic methods.

A fundamental data, obtained in this article is allowed us to use technology for crystallisation any materials (glass, quartz, semiconductors, whisker graphite).

The term "coherent nanowhiskerography" - is introduced for the first time by us, because the whisker crystallization occurs with a delay in reflection from two diffraction gratings, which are situated at the different depths. Thus, each whisker repeats in structure a retarded whisker during the crystalization. Due to this, unique optical and electrophysical properties arise.

Since at the ends of whiskers there is only 1-2 atoms with dangling chemical bonds, therefore, the technology is extremely promising for the new direction of creating a new direction of terahertz technology and also up to date generations of the computing technology.

Keywords: Whiskers, Polycarbonate, Crystallization, Laser Plasma, Interference, Light Dispersion, Coherence, Diffraction Grating

\section{Introduction}

First grown whiskers were obtained from silica on a catalyst in the form of gold seed drops according to the vapour-liquid-crystal mechanism (Dubrovskii, Sibirev, Harmand, \& Glas, 2008; Gorbik, Dubrovin, Demchenko, Filonenko, \& Dadykin, 2007; Ryabtsev, Kushchev, Soldatenko, Khadiya, Popov, \& Domashevskaya, 2011; Severyukhin \& Severyukhina, 2011; Givargizov \& Chernov, 1973). There is also a mechanism without a liquid phase vapour-liquid-crystal (Ryabtsev, Kushchev, Soldatenko, Khadiya, Popov, \& Domashevskaya, 2011; Givargizov, 1977).

Presently, there is no united theory of whisker growth; however, there are convincing approaches to modeling this process in particular cases. A model of whisker growth according to this mechanism is mathematically described in (Dubrovskii, Sibirev, Harmand, \& Glas, 2008; Severyukhin \& Severyukhina, 2011). In classical works by E. I. Givargizov (Givargizov \& Chernov, 1973; Givargizov, 1977) it is emphasized that the growth occurs due to a chemical potential difference between a metal drop and liquid phase of growing substance, which causes migration of silica atoms to the point of growth. 
According to this model, the rate of growth is described by the expression $V_{L}=K\left[\Delta \mu^{0}-\frac{R_{0}}{R}\right]^{2}$, where $\Delta \mu^{0}$ is the chemical potential difference between the vapour and crystal phases, $K$ is the kinetic coefficient of crystallization, $R$ is the radius of a whisker crystal, $\# R_{0}$ is the characteristic Givargizov-Chernov dimension.

The same basic mechanism acts when whiskers are grown by molecular epitaxy methods (Mamutin, 1999).

In a review devoted to the century anniversary of researches on oriented particle joining (Ivanov, Fedorov, Baranchikov, \& Osiko, 2014) it is stressed that nanowire growth occurs coherently. However, the abovementioned papers consider whisker growth, which occurs slowly and in sufficiently equilibrium conditions.

In the present work, we suggest a new approach to the growth of coherent whiskers with sharp tips by laser action on a plastic composite in strongly non-equilibrium conditions (short-time and quickly varying gradients of temperature and electromagnetic fields of a laser intensity.

Recently, it was found that under the action of a pulsed laser radiation on solutions of metal salts in a paper matrix in the irradiation zone at a temperature of $\sim 3300^{\circ} \mathrm{C}$, metal reduction and crystallization is observed with nanocrystal origin at a rate of $80-100 \mathrm{~m} / \mathrm{s}$ (Bogonosov \& Maksimovskii, 2011). The phenomenon observed was termed the high-rate metal nanocrystallization in laser plasma (Bogonosov \& Maksimovskii, 2011). The phenomenon is based on the employment of the following effects: the self-focusing, light-hydraulic effect, particle acceleration in a laser beam (Askaryan, 1973; Askaryan \& Mukhamedzhanov, 1981; Lugovoi \& Prokhorov, 1973), effect of sublimation evaporation (Askaryan \& Moroz, 1962), laser beam splitting, which were discovered at the P. N. Lebedev Physical Institute, RAS.

On a basis of this phenomenon, crystals of shattuckite were for the first time synthesized from copper compound solutions and silicon dioxide under the action of laser radiation (Maximovskii, Turyanskiy, Bogonosov, Gizha, Senkov, \& Pirshin, 2016). Attempts to artificially synthesize this mineral in other laboratories were not successful because it was impossible to produce pressure of above one thousand atmospheres. In shattuckite crystallization, the self-focusing of a laser beam occurred with the origin of moving focuses, which were seeds for starting crystallization with the following growth of similarly oriented crystals (Askaryan \& Mukhamedzhanov, 1981; Lugovoi \& Prokhorov, 1973).

Recently, Maksimovsky S.N. and co-workers (Maximovskii, Turyanskiy, Bogonosov, Gizha, Senkov, \& Pirshin, 2016) observed the effect of visible light dispersion after the interaction of a laser radiation with a polymer composite material comprised of a thin aluminum film, multilayer polycarbonate structure, and two diffraction gratings. However, the mechanism of laser radiation interaction with the polymer composite has not been studied in the patent (Maximovskii, Turyanskiy, Bogonosov, Gizha, Senkov, \& Pirshin, 2016).

According to works by Askar'yan (Maximovskii \& Radutskii, 2015) and Townes (Townes, 1964; Chiao, Garmie, \& Townes, 1964) concerning matter evaporation, the pressure produced by evaporated substance can be estimated by the formula $p \approx \frac{\alpha I v_{u}}{\lambda}$, where $\alpha$ is the part of energy consumed by the evaporation process (for powerful beams and absorbing substances of a target, it can approximately be taken unity (Maximovskii \& Radutskii, 2015; Chiao, Garmie, \& Townes, 1964)), $I$ - is the beam intensity (the density of energy flux), $v_{u}$ is the velocity of outgoing particles, which just produce the pressure, parameter $\lambda \sim \lambda_{0}+\frac{1}{2} v_{u}^{2}$ is the sum of the specific boiling heat for the material and the specific energy for material vapour acceleration.

The laser radiation parameters mentioned in the preceding works by Vodop'yanov (Vodop'yanov, Kozlov, Kucherenko, Maksimovskii, \& Radutskii, 2003) and other papers on synthesis of copper crystals (Bogonosov \& Maksimovskii, 2011) and shattuckite (Askaryan \& Moroz, 1962) are presented in Table 1. The parameters of plastic treatment are also given for comparison (Maximovsky, Stavtsev, \& Nedelkin, 2017). From the powers presented one can calculate the reached energy densities and pressures of light and evaporated substance. 
Table 1. Comparison of laser and crystallization parameters in various papers

\begin{tabular}{|c|c|c|c|}
\hline \multirow[t]{3}{*}{ Crystallised material } & \multirow{3}{*}{ 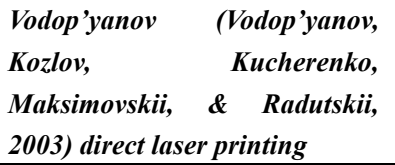 } & copper, shattuckite (Bogonosov & \multirow{3}{*}{$\begin{array}{l}\text { plastic (Maximovsky, } \\
\text { Stavtsev, \& Nedelkin, 2017) }\end{array}$} \\
\hline & & \& Maksimovskii, 2011; & \\
\hline & & Askaryan \& Moroz, 1962) & \\
\hline Laser type & Nd:YAG & Nd:YAG & Nd:YAG \\
\hline Wavelength & $1,06 \mu$ & $1,06 \mu$ & $1,06 \mu$ \\
\hline Power (average) & $4 \mathrm{~W}$ & $5 \mathrm{~W}$ & $20 \mathrm{~W}$ \\
\hline Pulse repetition frequency & $0-100 \mathrm{kHz}$ & $3 \mathrm{kHz}$ & $3 \mathrm{kHz}$ \\
\hline Pulse energy & $10 \mu \mathrm{J}$ & $1,7 \mathrm{~mJ}$ & $6,7 \mathrm{~mJ}$ \\
\hline Pulse duration & $150 \mathrm{~ns}$ & $13 \mathrm{~ms}$ & $1 \mu \mathrm{s}$ \\
\hline Pulse power & $267 \mathrm{~W}$ & $128 \mathrm{~W}$ & $6667 \mathrm{~W}$ \\
\hline Spot size & $30 \mu$ & $10 \mu$ & $30 \mu$ \\
\hline Energy flux density & $3,77 * 10^{11} \mathrm{~W} / \mathrm{m}^{2}$ & $1,67 * 10^{12} \mathrm{~W} / \mathrm{m}^{2}$ & $9,43 * 10^{12} \mathrm{~W} / \mathrm{m}^{2}$ \\
\hline Light pressure, $\mathrm{Pa}$ & 1888 & 8167 & 47190 \\
\hline Vapour pressure, atm. & 797 & 3450 & 19936 \\
\hline
\end{tabular}

Thus, the pressure of light radiation in the laser operation regimes considered in (Bogonosov \& Maksimovskii, 2011; Askaryan \& Moroz, 1962; Vodop'yanov, Kozlov, Kucherenko, Maksimovskii, \& Radutskii, 2003) reaches fractions of atmosphere, and vapour reactive pressure may reach thousands atmospheres, which corresponds to a pressure difference by several orders between pressures of light and evaporated substance (Maximovskii \& Radutskii, 2015).

\section{Experments}

In the present work, we investigate this phenomenon in more details by studying the action of a pulsed laser radiation on the composite polymer substrates, which comprise two diffraction gratings in a polycarbonate layer based on 2,2-bis(4-oxyphenyl)propane. The widths of both the polycarbonate layers are $0.6 \mathrm{~mm}$, and a $5 \mu$ distance between the gratings is filled with an optically transparent glue. The diffraction grating period is $0.74 \mu$. For substrate samples was taken a DVD-disk with the surfaces deposited by an aluminum foil layer (Figure 1).

A neodymium laser with a wavelength of $1.06 \mu \mathrm{m}$ used in this work operated in a Q-switched pulse-periodic regime with a pulse repetition frequency of $3 \mathrm{kHz}$, average radiation power of $20 \mathrm{~W}$, and pulse duration of $\sim 1 \mu \mathrm{s}$. The beam spot diameter was about $100 \mu \mathrm{m}$, which can be estimated from the optical microscope photograph presented below.

We assumed that laser irradiation of such substrates may generate whiskers in the irradiated material zones, which seems promising for producing a texture picture by a laser beam.

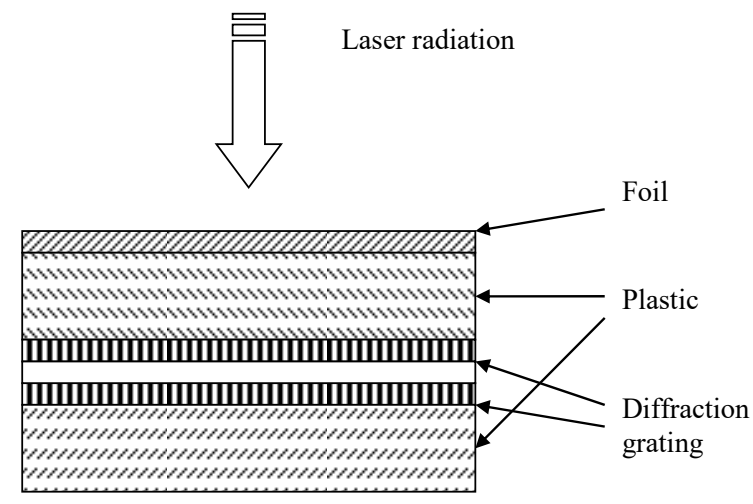

Figure 1. Schematic design of a composite material sample. The samples obtained were studied by optical microscopy and on a scanning probe mocroscope (ACM) Certus Light V produced by Nano Scan Tech 


\subsection{Research Methods}

Doubling of points produced by laser shots was observed in an optical microscope (Figure 2). On can see that the points are ordered reproducible formations in the irradiated zone in the form of doubled channels each of size $0.2-0.3 \mathrm{~mm}$. Importantly, the channels have similar shapes and equal separations between them. Channel surfaces and structure were more thoroughly studied with atomic-force microscopy. A relief of a channel bottom and space between the points was investigated. The results are presented in Figure 3.

From data of atomic-force microscopy (Figure 3), it follows that a boundary zone "a" between crystals is a specific array of whiskers of height $0.5-1 \mu \mathrm{m}$ with nano-size tips. A dimension of the largest formations reaches $3 \mu \mathrm{m}$.

Possibly, this "pale" characterises a remained crystal structure of polycarbonate surface after the action of a laser pulse. Interestingly, not only the size and shape of channels produced by laser shots are symmetrical relative to the zone of the boundary pale, but also the array of peaks on the surface is not arranged chaotically, but has a noticeable symmetry relative to the boundary pale (for example, zone "b" in Figure 3), thus, producing a mirror picture.

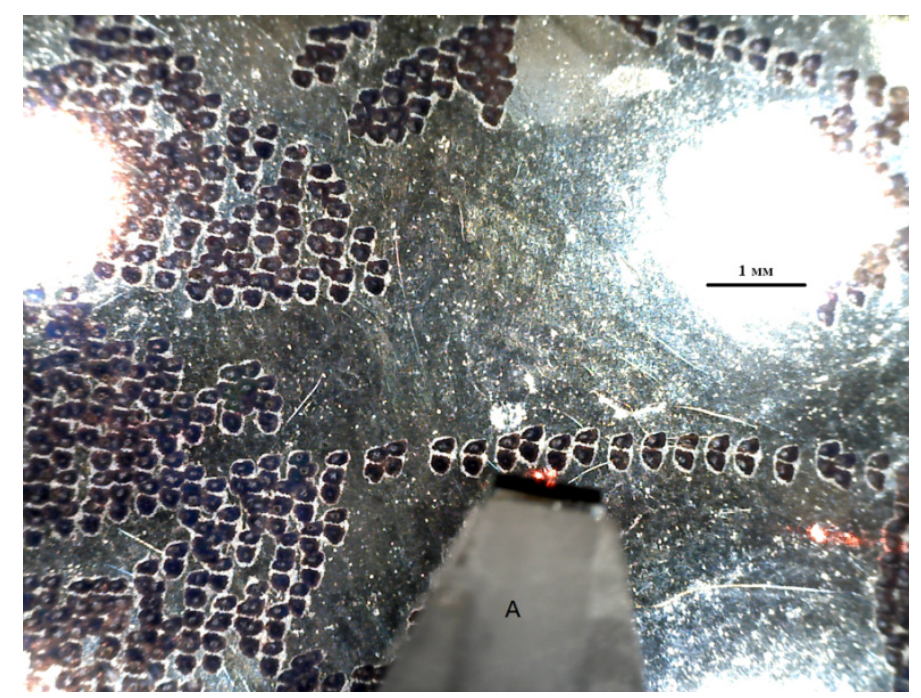

Figure 2. Sample surface after laser irradiation (optical microscope). One can see a probe of the atomic-force microscope (A)

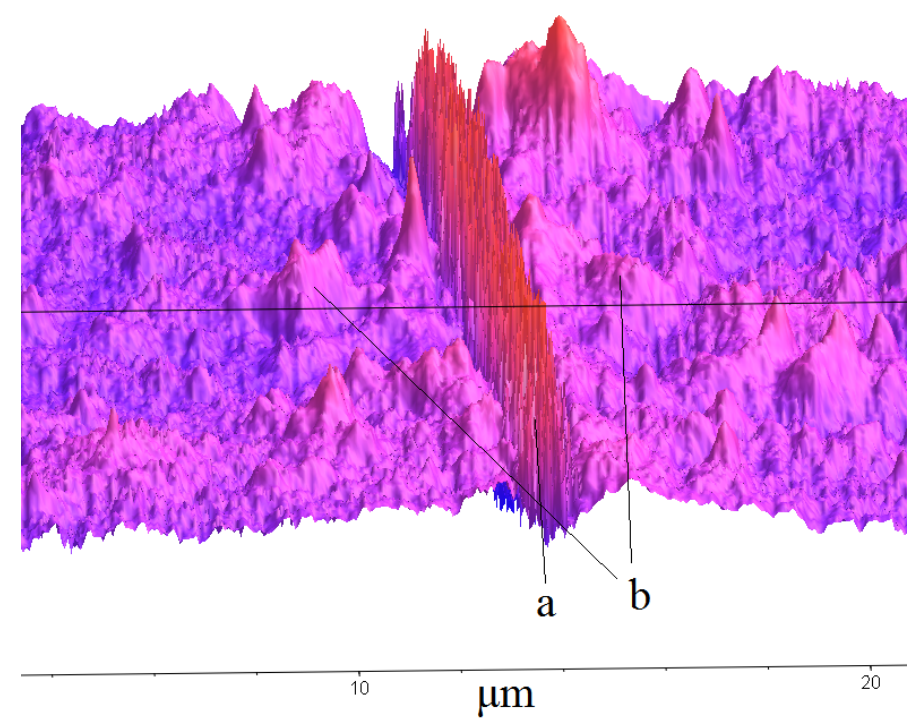

Figure 3. Surface structure: "a" - whiskers in the boundary zone; "b" - a symmetrical zone on a surface (atomic-force microscope) 

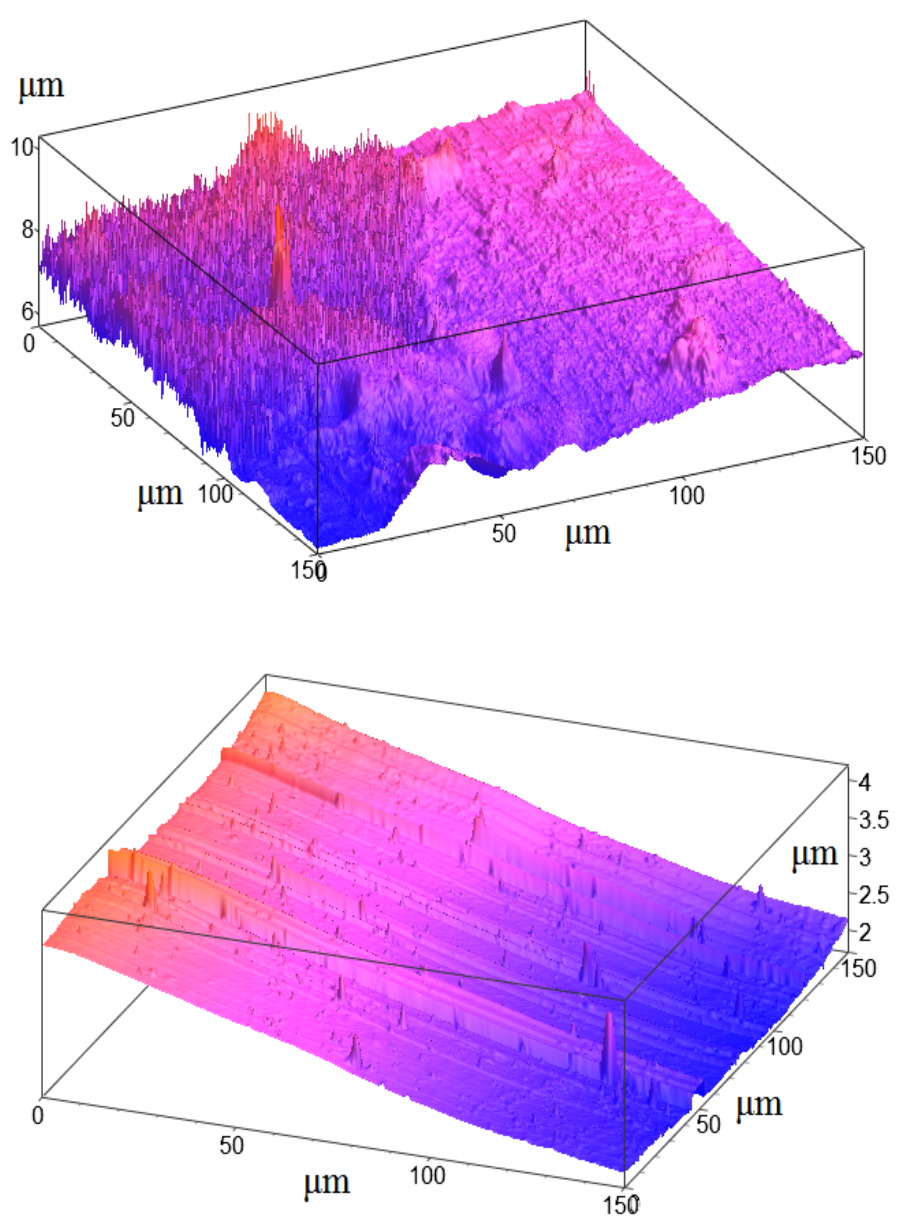

For explaining the origin of a symmetrical structure on a polycarbonate surface, we assumed that a focused laser beam having reached two diffraction gratings reflects from them in two beams with an optical path difference between them, destroys the grating in the beam zone, and causes the material to melt with the following vitrification of the polycarbonate melt in two symmetrical ordered channels.

\subsection{Heat Model}

Photons of laser emission that have attained the foil of a composite material destroy an aluminum crystal lattice (Figure 4,a) and embed free electrons and aluminum atoms in polycarbonate (Figure 4,b) (Maximovskii \& Radutskii, 2015; Menushenkov, Nevolin, \& Petrovskii, 2010). This causes instantaneous melting of polycarbonate in the irradiated channels (Figure 4,c-f). The melting temperature of crystalline polycarbonate is $223-225^{\circ} \mathrm{C}$ (Smirnova \& Erofeeva, 1975; Encyclopedia of Polymers, 1974).

The "pipe" rays (with a reduced intensity at the beam centre) are produced just in the self-focusing process. Beams of a sub-threshold power split to the threshold-power beams according to Towns, which initiate the growth of whiskers. This explains the highest whisker concentration at beam boundaries and, especially, between the doubled points.

Heat distribution in a polymer material was estimated by using the heat conduction equation. In the one-dimensional case, the problem is solved in the range $0<x<l$ and $0<t<T$, (where $x$ is the coordinate along the sample depth, $l$ is the sample length, $T$ is the laser pulse duration), $a^{2}$ is a positive constant (thermal diffusivity), $u$ is the temperature, and $t$ is time:

$$
\frac{\partial u}{\partial t}-a^{2} \frac{\partial^{2} u}{\partial x^{2}}=0
$$

The boundary conditions are: 


$$
\begin{gathered}
\frac{\partial u(0, t)}{\partial x}=-\frac{q_{0}}{k} \\
u(l, t)=u_{0}
\end{gathered}
$$

From the side of radiation input $x=0$ in the result of its transfer to heat there will be a heat flow $q_{0}$ (the second-kind boundary condition); $k$ is the heat-conduction coefficient. From the other side, the temperature of environment $u_{0}$ is maintained (the first-kind boundary condition). For a time lapse of the laser pulse action, the boundary $x=l$ can be considered as a thermostat (isothermal boundary). In experiments, the sample is pressed to a massive aluminum table, which possesses high heat conductivity. Prior to the laser irradiation, the initial temperature of the substrate coincides with the environment temperature $u_{0} u(x, 0)=u_{0}$.

The Sturm-Liouville problem stated in this way was solved by the Fourier method. The following solution was obtained:

$$
u(x, t)=u_{0}+\frac{q_{0}}{k}(l-x)-\frac{8 q_{0} l}{\pi^{2} k} \sum_{n=0}^{\infty} \frac{1}{(2 n+1)^{2}} \cos \left[\left(\frac{\pi}{2 l}+\frac{\pi n}{l}\right) x\right] \exp \left\{-a^{2}\left(\frac{\pi}{2 l}+\frac{\pi n}{l}\right)^{2} t\right\}
$$

where $n=0,1,2,3 \ldots$

Calculations based on this solution show that for the time lapse of laser pulse action, heat penetrates polycarbonate by the heat conduction mechanism to a depth of at most $2 \mu \mathrm{m}$. Such parameters as the laser pulse duration, polycarbonate characteristics, and energy flow density were used in the calculation, and the pulse shape was a rectangular step. In this case, the metal foil was a source of heat, because polycarbonate is transparent at the laser radiation wavelength.

Thus, the laser pulse energy is distributed in a near-surface layer of the material. Then the heat dissipates inside the substrate to a depth of $40-50 \mu \mathrm{m}$ by the heat conduction mechanism, because convective streams in a plastic melt have no time to arise due to a high viscosity of the melt (Smirnova \& Erofeeva, 1975; Encyclopedia of Polymers, 1974; Shnell, 1967).

After vitrification of the polycarbonate melt, symmetrical ordered structures are observed on a sample surface in the irradiation zones (Figures 2, 3). In scanning the sample surface by a laser beam, these structures produce an original image: the images are transparent in the visible range, and in reflection the images exhibit well pronounced dispersion when the angle of illumination by white light is varied (Figure 5).

The seed metal drops arise in a melting foil on a sample surface, from which whiskers grow. Drop dimensions are from 0.5 to $2 \mu \mathrm{m}$ (the FWHM value); inside a pale, at the boundary between double points the whisker FWHM is $0.3 \mu \mathrm{m}$. The whisker height varies from 0.2 to $1 \mu \mathrm{m}$.

\section{Discussion of the Results}

In the work by Givargizov it was pointed that the growth of whiskers occurs due to the chemical potential difference. In our case, this difference is realised due to a transfer of laser radiation energy to a substrate, which changes the chemical potential of the latter. Authors of (Givargizov, 1977) also inform that drops arise at wisker ends in a result of the growth, and it is necessary to remove and sharpen the wiskers by chemical etching for applications in electronic devices. In the present work, whiskers with sharp tips are produced just in the process of growing.

In contrast to classical mechanisms, where whiskers sufficiently slowly grow in equilibrium conditions, in this work whiskers are formed for a time lapse of laser pulse duration in strongly non-equilibrium conditions. 


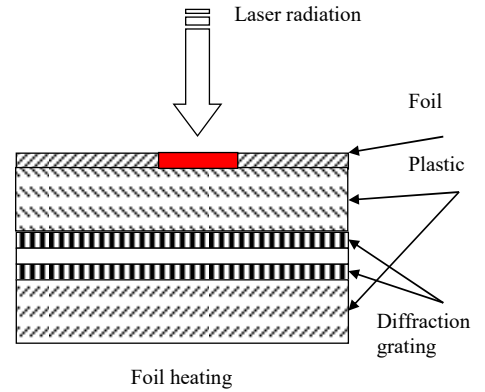

A)

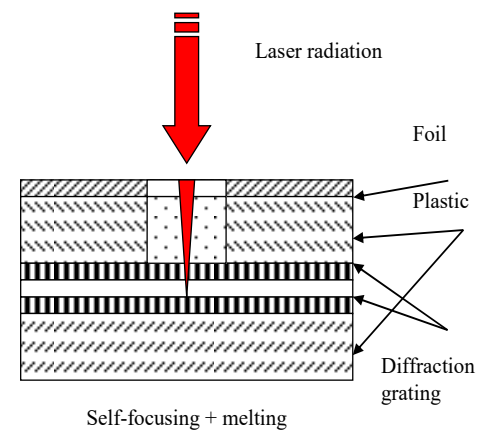

C)

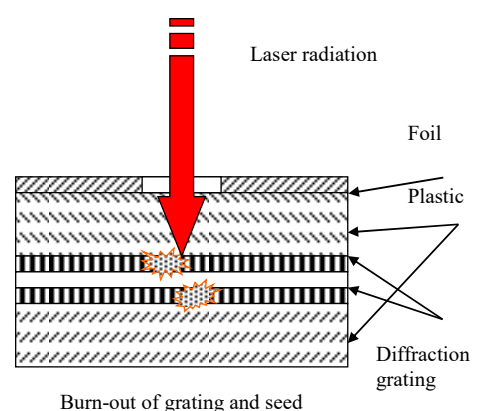

E)

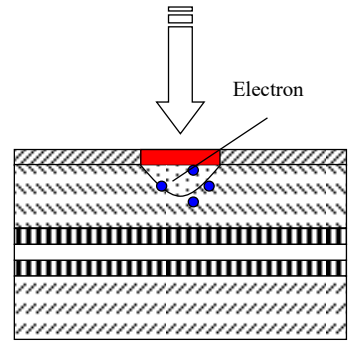

Electron heat emission

B)

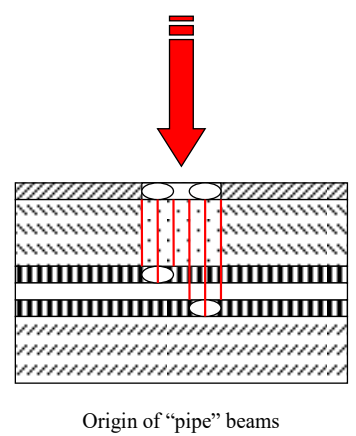

D)

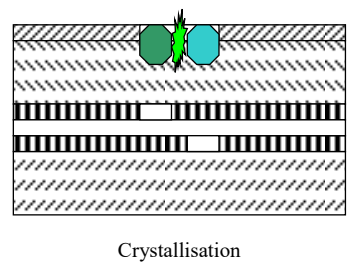

F)

Figure 4. Schematic diagram of processes occurring in studied composition based on polycarbonate under pulsed laser irradiation 


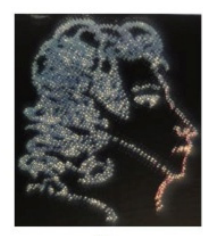

a

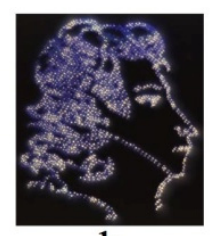

b

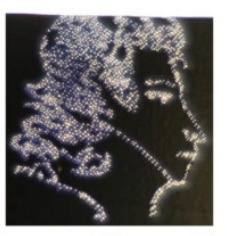

$\mathrm{c}$

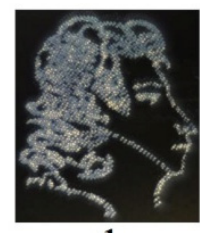

d

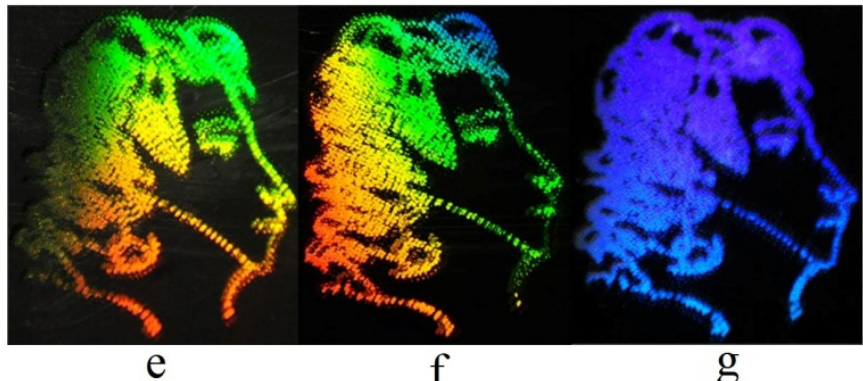

g

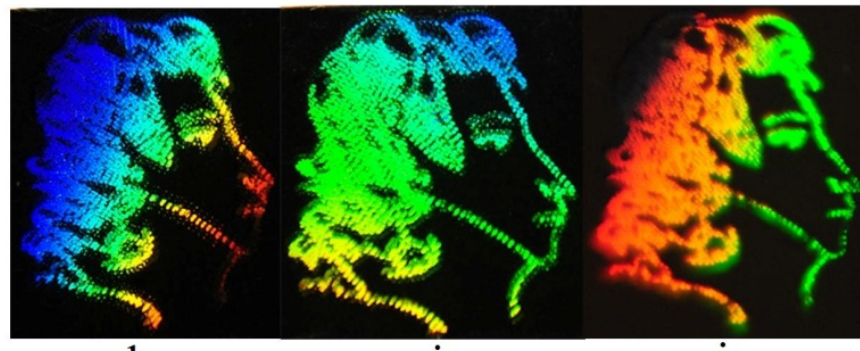

$\mathrm{h}$

i

j

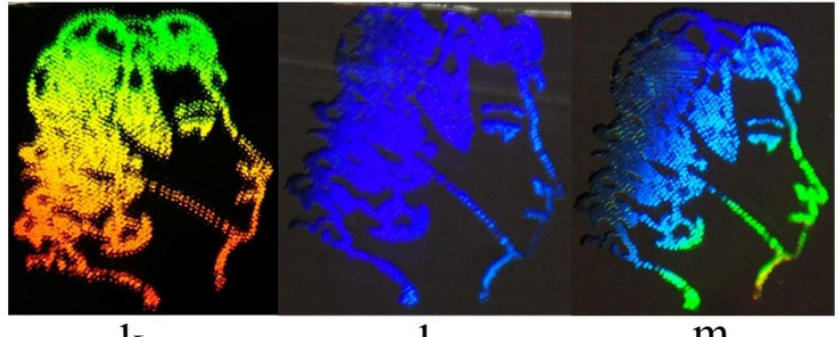

$\mathrm{k}$

1

$\mathrm{m}$

Figure 5. Visible optical properties of obtained images: a-d transparent in transmitted light; e-l colour image in reflected light at various angles of illumination

\section{Conclusion}

From data of atomic-force microscopy it follows that the highest concentration of whiskers in the form of a pale is observed at the boundary of points produced by a laser spot and at the boundary between doubled points. This fact can be explained by that in a result of the self-focusing, a "pipe" beam may be produced with a lower intensity at the beam centre (Askaryan, Studentov, \& Chistyi, 1970). The intensity of laser radiation at the beam boundary exceeds the threshold power; hence, the beam splits to beams of the threshold power (Chiao, Garmie, \& Townes, 1964) along which whiskers grow.

Just the fast origin of such secondary structures in irradiated polycarbonate samples may be responsible for the ordering observed in the irradiation zone. The initial polycarbonate with diffraction gratings possesses dispersion properties; however, light dispersion in the irradiation zone is much brighter, which makes it possible to form images in the irradiation zone.

The observed light dispersion in polycarbonate samples (Figure 5) is interesting for depositing protective images capable of changing the colour versus the illumination angle.

This technological direction is rather perspective for crystallization of the battery electrodes (silicon anodes), automotive catalysts, battery for space (silicon, and $\mathrm{A}_{3} \mathrm{~B}_{5}$ compounds), solar cells. 
We can reproduce the same facilities in the ehin films (Maximovsky, Stavtsev, \& Nedelkin, 2017; Maximovsky, Ivanova, \& Stavtsev, 2018) as well as crystallize silicon and dislocation-free whisker carbon, which have higher strength and Young's modulus.

This technology allows us to provide cocrystallization of any materials due to hight temperature and pressure. The same processes we can observe in the Earth's bowels. The confirmation of this processes is published in (Maximovskii, Turyanskiy, Bogonosov, Gizha, Senkov, \& Pirshin, 2016).

The work was performed in the frameworks of the State task for scientific-research work 16.9298.2017/5.1 on topic "Study of crystallization processes of plastics and composites under an action of a powerful laser radiation" of the Ministry of Education and Science, RAS.

\section{Conflict of interests}

The authors declare that there is no conflict of interests regarding the publication of this paper.

\section{References}

Askaryan, G. A. (1973). Uspekhi Fiz. Nauk, 111(2), 249.

Askaryan, G. A., \& Moroz, E. P. (1962). Zh. Exp. Teor. Fiz., 43(6), 2319.

Askaryan, G. A., \& Mukhamedzhanov, M. A. (1981). Pis'ma ZhETF, 33(1), 48.

Askaryan, G. A., Studentov, V. B., \& Chistyi, I. L. (1970). Usp.Fiz.Nauk, 100(3), 519.

Bogonosov, K. A., \& Maksimovskii, S. N. (2011). Doklady Physics, 56(8), 419-422.

Chiao, R. Y., Garmie, E., \& Townes, C. H. (1964). Phys. Rev. Lett., 13, 479.

Dubrovskii, V. G., Sibirev, N. V., Harmand, J. C., \& Glas, F. (2008). Growth kinetics and crystal structure of semiconductor nanowires. Physical Review B, 78, 235301.

Entsiklopediya polimerov (Encyclopedia of Polymers) (1974). Moscow: Sov. Entsiklopediya, 2, 846.

Givargizov, E. I. (1977). Rost nitevidnykh i plastinchatykh kristallov iz para (Growth of thread-like and lamellar crystals from vapour) (p. 303). Moscow: Nauka.

Givargizov, E. I., \& Chernov, A. A. (1973). Kristallografiya, 18(1), 147.

Gorbik, P. P., Dubrovin, I. V., Demchenko, Y. A., Filonenko, M. N., \& Dadykin, A. A. (2007). Khimiya, fizika $i$ tekhnologiya poverhnosti, 13, 294.

Ivanov, V. K., Fedorov, P. P., Baranchikov, A. E., \& Osiko, V. V. (2014). Uspekhi Khimii, 83(12), 1204.

Lugovoi, V. N., \& Prokhorov, A. M. (1973). Usp. Fiz. Nauk, 111(2), 203.

Mamutin, V. V. (1999). Pis'ma Zh. Teor. Fiz., 25(18), 55-63.

Maximovskii, S. N., \& Radutskii, G. A. (2015). Sposob usileniya svecheniya izobrazheniya, perelivayushchegosya tsvetami radugi, sozdannogo $v$ tele listovogo materiala. Patent of the Russian Federation No. 2537837. Byulleten' Izobretenii, No. 1.

Maximovskii, S., Turyanskiy, A., Bogonosov, K., Gizha, S., Senkov, V., \& Pirshin, I. (2016). Shattuckite Synthesis and the Pattern Formation by the Scanning Laser Beam. Tecnol. Metal. Mater. Miner, San Paulo, 13(3), 248-251.

Maximovsky, S. N., Ivanova, V. N., \& Stavtsev, A. Y. (2018). Nanowhiskerography - a New Method of Security Printing using Scanning Laser Radiation. Bulletin of the Lebedev Physics Institute, 45(11), 341-345. (C) Allerton Press, Inc.

Maximovsky, S. N., Stavtsev, A. Y., \& Nedelkin, V. I. (2017). Interaction of Pulsed Laser Radiation with a Polycarbonate-Based Composite. Bulletin of the Lebedev Physics Institute, 44(12), 374-379. Allerton Press, Inc.

Menushenkov, A. P., Nevolin, V. N., \& Petrovskii, V. N. (2010). Fizicheskie osnovy lazernoi tekhnologii (p. 212). Moscow: National Research Nuclear University "MEPHI".

Ryabtsev, S. V., Kushchev, S. B., Soldatenko, S. A., Khadiya, N. M. A., Popov, A. E., \& Domashevskaya, E. P. (2011). Kondensirovannye sredy i mezhfaznye granitsy, 13(1), 80-88.

Severyukhin, A. V., \& Severyukhina, J. Y. (2011). Izvestiya Tul'skogo gosudarstvennogo universiteta. Estestvennye nauki, (2), 267. 
Shnell, G. (1967). Khimiya i Fizika polikarbonatov (Chemistry and Physics of Polycarbonates) (p. 146). Moscow: Khimiya.

Smirnova, O. V., \& Erofeeva, S. B. (1975). Polikarbonaty (Polycarbonates) (p. 290). Moscow: Khimiya.

Townes, C. H. (1964). Phys Letter, 13, 479. The structure laser light.

Vodop'yanov, L. K., Kozlov, P. S., Kucherenko, I. V., Maksimovskii, S. N., \& Radutskii, G. A. (2003). Studying the Possibility of Applying the Light-Hydraulic Effect to Digital Printing Instruments and Experimental Techniques, 46(4), 549-553.

\section{Copyrights}

Copyright for this article is retained by the author(s), with first publication rights granted to the journal.

This is an open-access article distributed under the terms and conditions of the Creative Commons Attribution license (http://creativecommons.org/licenses/by/4.0/). 